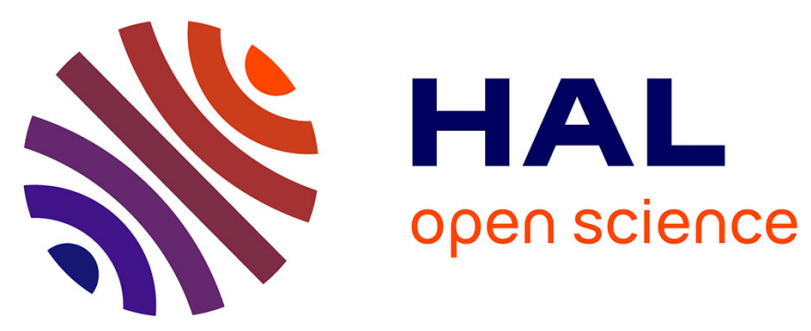

\title{
Band offsets in strained InGaAsP/InGaAsP quantum well optical modulator structures
}

Roland Martin, S. Wong, R. Nicholas, A. Smith, M. Gibbon, E. Thrush, J. Stagg

\section{- To cite this version:}

Roland Martin, S. Wong, R. Nicholas, A. Smith, M. Gibbon, et al.. Band offsets in strained InGaAsP/InGaAsP quantum well optical modulator structures. Journal de Physique IV Proceedings, 1993, 03 (C5), pp.C5-327-C5-330. 10.1051/jp4:1993567 • jpa-00251654

\section{HAL Id: jpa-00251654 https://hal.science/jpa-00251654}

Submitted on 1 Jan 1993

HAL is a multi-disciplinary open access archive for the deposit and dissemination of scientific research documents, whether they are published or not. The documents may come from teaching and research institutions in France or abroad, or from public or private research centers.
L'archive ouverte pluridisciplinaire $\mathbf{H A L}$, est destinée au dépôt et à la diffusion de documents scientifiques de niveau recherche, publiés ou non, émanant des établissements d'enseignement et de recherche français ou étrangers, des laboratoires publics ou privés. 


\title{
Band offsets in strained InGaAsP/InGaAsP quantum well optical modulator structures
}

\author{
R.W. MARTIN, S.L. WONG, R.J. NICHOLAS, A. SMITH*, M.A. GIBBON* ${ }^{*}$ E.J. THRUSH* and \\ J.P. STAGG*
}

Department of Physics, Clarendon Laboratory, Parks Road, Oxford OX1 3PU, U.K.

${ }^{*}$ BNR Europe Ltd., London Road, Harlow, Essex CM179NA, U.K.

\begin{abstract}
Magneto-absorption experiments on a range on $\operatorname{In}_{1-x} \mathrm{Ga}_{\mathbf{x}} \mathrm{As}_{\mathrm{y}} \mathrm{P}_{1-\mathrm{y}} /$ InGaAsP multi-quantum well (MQW) structures, including both tensile and compressive strained wells, are presented. Estimates for the band offsets are made in a lattice matched and a strain-balanced structure, and a model to predict the band offsets as a function of strain in InGaAsP heterostructures is described.
\end{abstract}

\section{Introduction}

Optoelectronic devices, such as lasers and modulators, employing InGaAsP MQW structures are attractive for operation in the important $1.55 \mu \mathrm{m}$ region. The benefits of strain in quantum well lasers are well known, related to modifications of the band structure, but in this paper advantages of introducing strain in optical electroabsorption modulators are discussed. The desirability of monolithically integrating a MQW laser and modulator make any advantages for strain in MQW modulators especially noteworthy.

\section{Advantages of strained layer electroabsorption modulators}

Electroabsorption modulators, based on the quantum confined Stark effect, have been demonstrated in a range of lattice matched combinations of InGaAsP based materials for $1.55 \mu \mathrm{m}$ operation. However, such devices suffer from sensitivity to the input polarisation and a tendency to saturate as the light intensity increases. Improved modulator performance, along with the possibility of polarization insensitive behaviour, might be achieved by using strain to engineer a co-incidence of the heavy and light hole excitonic transitions in the quantum wells of these devices. The absorption strength of this combined peak will be enhanced by the increased reduced exciton masses at this point, caused by coupling of the hole levels. However when quantum wells are placed under strain the confinement barriers for both heavy and light holes are expected to change. This in turn changes the rate at which holes escape from the wells in an electric field and thus alters the saturation behaviour of modulators. Consequently a knowledge of how the band offset ratio varies as a function of the strain and composition in a InGaAsP/InGaAsP quantum well is required.

The band offset ratios have been measured for a number of lattice matched $\operatorname{In}_{1-x} \mathrm{Ga}_{\mathbf{x}} \mathrm{As}_{\mathbf{y}} \mathrm{P}_{1-\mathrm{y}} / \mathrm{InP}$ combinations $^{(1)}$ but little is known about how they vary as strain is included and/or InGaAsP barriers used. Here we present the first measurements of the band offsets in InGaAsP/InGaAsP heterostructures and go on to consider a model to predict the band profiles as a function of strain.

3. Experimental results.

A number of $\mathrm{In}_{1-\mathrm{x}} \mathrm{Ga}_{\mathrm{x}} \mathrm{As}_{\mathrm{y}} \mathrm{P}_{1-\mathrm{y}} / \mathrm{InGaAsP} \mathrm{MQW}$ structures have been grown by low pressure MOVPE on InP substrates, including three lattice matched 50 well structures with $\lambda_{\mathrm{RT}}=1.58 \mu \mathrm{m}$ InGaAsP wells and $\lambda_{\mathrm{RT}}=1.18 \mu \mathrm{m}$ InGaAsP barriers. Measurements have also been made on a 6 well sample with $\lambda_{\mathrm{RT}}=1.58 \mu \mathrm{m}$ InGaAsP wells under $1 \%$ compressive strain $\left(\varepsilon_{\text {well }}=-1.0 \%\right)$ and $\lambda_{\mathrm{RT}}=1.25 \mu \mathrm{m}$ InGaAsP barriers under $1 \%$ tensile strain $\left(\varepsilon_{\text {barrier }}=+1.0 \%\right)$, and on a series of 4 period MQW with tensile strained $\operatorname{In}_{1-\mathrm{x}} \mathrm{Ga}_{\mathrm{x}}$ As wells 
and lattice matched $\lambda_{\mathrm{RT}}=1.17 \mu \mathrm{m}$ InGaAsP barriers.

Interband magneto-optical measurement have been performed in magnetic fields up to $15 \mathrm{~T}$ at $4.2 \mathrm{~K}$. The absorption coefficient of typical lattice matched (E593), compressively strained (E863, $\varepsilon_{\text {well }}=-1 \%$, $\varepsilon_{\text {barrier }}=+1 \%$ ) and tensile strained (E866 with $\varepsilon_{\text {barrier }}=+0.3 \%$, E 870 with $\varepsilon_{\text {barrier }}=+1.0 \%$ ) samples are shown in Fig. 1(a) at zero magnetic field. Maxima are resolved for E1-HH1, E1-LH1, and E2-HH2 excitonic transitions, with higher levels resolved in some cases, along with a bulk transition from the quaternary buffer layers. The transitions observed in the series of tensile strained samples are plotted in Fig. 1(b), and the E1-HH1 and E1-LH1 splitting is seen to be roughly proportional to $\varepsilon_{\text {well }}$. The magnetic field improves the resolution of the transitions and leads to one or more series of Landau levels originating from the E1-HH1 and/or E1-LH1 transition. This is illustrated in Figs. 2(a,b), which show the excitonic transitions observed in E863 and E870 as a function of magnetic field.
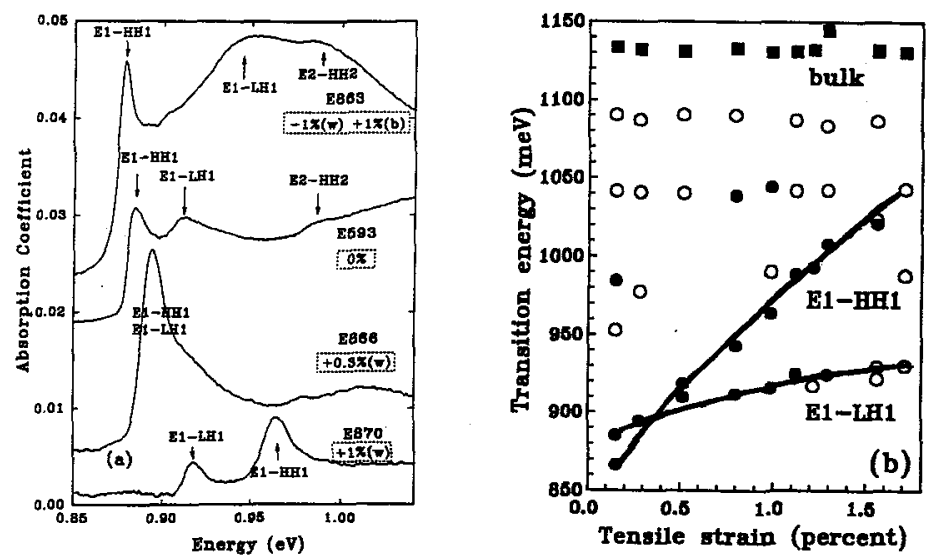

Figure 1: (a) Absorption coefficient per well at $4 \mathrm{~K}$ and $0 \mathrm{~T}$. The origins of the spectra are shifted for clarity. (b) The observed transitions in the tensile strained MQW as a function of the measured strain in the well. The solid circles show strong transitions, the open circles weak or broad features.


Figure 2: (a) The observed excitonic transitions as a function of magnetic field for E863, with compressively strained wells and tensile strained barriers. (b) As (a) but for E870.

For sample E863, with compressive strained wells, a clear E1-HH1 transition and associated Landau levels are resolved and there are two weak transitions at higher energy. These are expected to be the E1-LH1 and E2-HH2, respectively, and it is seen that the strain has generated a splitting of over $60 \mathrm{meV}$ between the heavy and light hole levels. For the lattice matched sample, E593, the E1-HH1, E1-LH1, and $\mathrm{E} 2-\mathrm{HH} 2$ transitions are resolved and the heavy hole levels appears $27 \mathrm{meV}$ below the light hole. With tensile strain in the well a co-incidence of the E1-LH1 and E1-HH1 transitions is possible, as has been achieved for 
E866, with $\varepsilon_{\text {well }}=+0.3 \%$. For larger tensile strains the lowest transition is E1-LH1 as seen in Sample E870, with $\varepsilon_{\text {well }}=+1.0 \%$ strain. Landau levels from both the E1-HH1 and E1-LH1 levels can be resolved in the tensile strained samples, but those of the light hole level dominate, even though the intensity of the E1-LH1 is weaker than that of $\mathrm{E} 1-\mathrm{HH} 1$ at $0 \mathrm{~T}$.

The energies of the observed transitions are fitted using a 3-band k.p perturbation theory, including far-band terms, within the envelope function approximation. A similar approach to magneto-absorption data from a lattice matched InGaAs/InGaAsP MQW has been used to give an accurate estimate of the band discontinuities $\left(\Delta \mathrm{E}_{\mathrm{c}}=0.38 \Delta \mathrm{E}_{\mathrm{g}}\right.$ ), due to the observation of excitonic transitions from both confined hole levels and valence band continuum states to confined electron levels ${ }^{(2)}$. In the present samples transitions from bound to continuum states are not resolved but a fitting to the observed transitions enables a less accurate estimate of the band offsets to be made.

For the case of the lattice matched $\lambda_{\mathrm{RT}}=1.58 \mu \mathrm{m}$ InGaAsP / $\lambda_{\mathrm{RT}}=1.18 \mu \mathrm{m}$ InGaAsP samples the E1HH1, E1-LH1, and E2-HH2 levels are fitted as a function of the band offset ratio for a range of layer thicknesses (whilst maintaining the period measured by X-ray.) This is shown in Fig. 3(a) which also shows the energies of the observed transitions, along with the estimated errors in the measurement. It is seen that, with the assumption that the ratio of well and barrier thicknesses are within $5 \%$ of the nominal value, $\Delta \mathrm{E}_{\mathrm{c}} / \Delta \mathrm{E}_{g}=0.40 \pm 0.04$ for this combination. Along with measurements on various compositions of InGaAsP lattice matched to $\operatorname{InP} P^{(1)}$, and on a lattice matched InGaAs/InGaAsP MQW(1) this result indicates that $\Delta \mathrm{E}_{c} / \Delta \mathrm{E}_{g}$ remains close to 0.4 for all the InGaAsP based lattice matched MQW measured thus far.
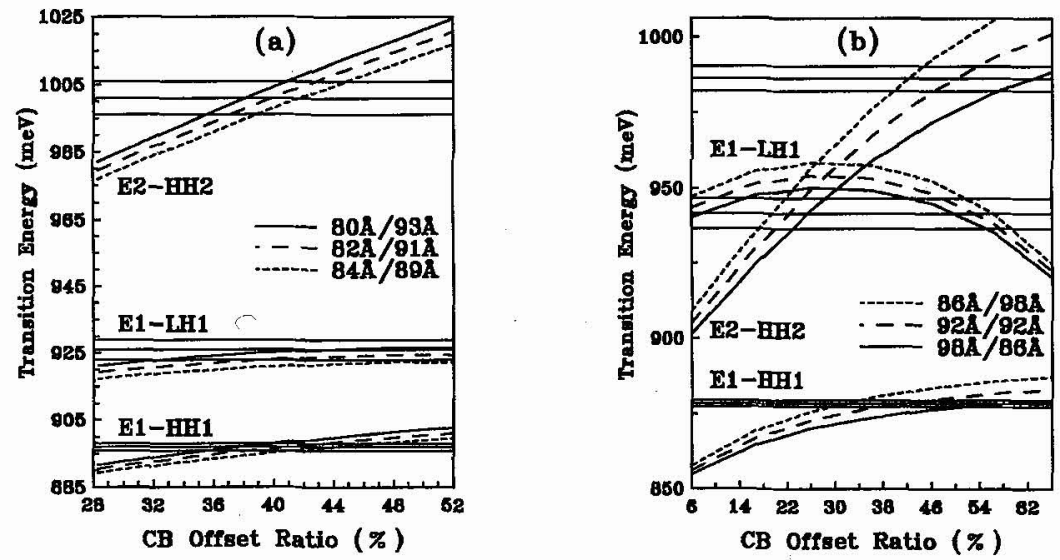

Figure 3: (a) The calculated and observed excitonic transitions as a function of the band offset ratio and layer thicknesses in a lattice matched $\lambda_{\mathrm{RT}}=1.58 \mu \mathrm{m}$ InGaAsP $/ \lambda_{\mathrm{RT}}=1.18 \mu \mathrm{m}$ InGaAsP sample. (b) As (a) but for a $\lambda_{\mathrm{RT}}=1.58 \mu \mathrm{m}, \varepsilon=-1 \%$ In GaAsP well with $\lambda_{\mathrm{RT}}=1.25 \mu \mathrm{m}, \varepsilon=+1 \%$ InGaAsP barriers.

The above approach is now used for $\mathrm{E} 863\left(\varepsilon_{\text {well }}=-1 \%, \varepsilon_{\text {barrier }}=+1 \%\right)$ for which the X-ray data resolved a slight uncompensated compressive strain along with a MQW period of $184 \AA$. The layer compositions are established from bulk calibration runs, although the limit to the thickness of such layers leads to greater uncertainties in the growth rates, than for lattice matched layers. Fig. 3(b) shows the three transitions observed in E863 (with estimated errors), along with the fitted values, and shows that the splitting of the E1-LH1 and E2-HH2 provides a sensitive measure of the band offset ratio. The fit, after a very slight adjustment to the nominal compositions, indicates that $\Delta \mathrm{E}_{c} / \Delta \mathrm{E}_{g}=0.52 \pm 0.05$. For the tensile strained samples the lack of higher transitions which can be positively identified renders the above approach unwise.

\section{Modelling of band offsets.}

Our model for estimating band offsets as a function of strain is based on the 'model-solid' approach of Van de Walle ${ }^{(3)}$. This calcnlates changes in relative energy levels, using deformation potentials to account for strain effects. The energy of the average valence band position for the bulk semiconductor materials, $\mathrm{E}_{v, a v}$, is required, for which values have been calculated for the III-V binary compounds ${ }^{(3)}$. When considering InGaAsP structures it is necessary to develop the correct interpolation scheme for calculating the values of $\mathrm{E}_{v, a v}$, and other parameters, from those of the constituent binaries. One such scheme involves 
Table 1: Band offsets estimated at $4 \mathrm{~K}$ in a range of InGaAsP/InGaAsP heterostructures using the model solid theory. Column (a) uses interpolated $\mathrm{E}_{v, \text { av }}$ values, whilst (b) assumes an unstrained $\Delta \mathrm{E}_{c} / \Delta \mathrm{E}_{g}=0.40$.

\begin{tabular}{|c|c|c|c|c|c|c|c||c|c|c||}
\hline \multirow{2}{*}{ Sample } & \multicolumn{2}{|c|}{ Well material } & \multicolumn{2}{|c|}{ Barrier material } & \multicolumn{3}{c|}{ (a) (in meV) } & \multicolumn{3}{c|}{ (b) (in meV) } \\
\cline { 2 - 11 } & $\lambda_{\mathrm{RT}}(\mu \mathrm{m}$ & $\varepsilon(\%)$ & $\lambda_{\mathrm{RT}}(\mu \mathrm{m})$ & $\varepsilon(\%)$ & $\Delta \mathrm{E}_{e}$ & $\Delta \mathrm{E}_{h h}$ & $\Delta \mathrm{E}_{l h}$ & $\Delta \mathrm{E}_{e}$ & $\Delta \mathrm{E}_{h h}$ & $\Delta \mathrm{E}_{l h}$ \\
\hline \hline E863 & 1.58 & -1.0 & 1.25 & +1.0 & 110 & 201 & 62 & 25 & 285 & 147 \\
\hline E839/E593 & 1.58 & - & 1.18 & - & 95 & 182 & 183 & 112 & 166 & 167 \\
\hline E870 & 1.45 & +1.0 & 1.17 & - & 77 & 148 & 223 & 144 & 81 & 156 \\
\hline
\end{tabular}

bowing parameters calculated using the hydrostatic deformation potentials and lattice parameters of the constituents ${ }^{(4)}$. However, this assumption may prove inaccurate for some InGaAsP compositions, and is found to give poor agreement with the values measured in the lattice matched ternary/quaternary and quaternary/quaternary combinations described above. An alternative assumption, based on the fact that all measurements in unstrained InGaAsP systems have returned a value for $\Delta \mathrm{E}_{c} / \Delta \mathrm{E}_{g}$ close to 0.4 , is to arbitrarily assign a $40 / 60$ split of the total unstrained band discontinuity for an InGaAsP heterostructure, and then apply the effects of strain using the model solid theory. This should be reasonably accurate for small strains, say $\pm 0.5 \%$ but will become increasingly inaccurate thereafter.

The remaining input parameters are obtained by interpolation of the binary values as in Ref. 4, with the exception of a quaternary bowing factor used in the interpolations of the energy gap and spin orbit splitting ${ }^{(5)}$. Table 1 compares the band offsets predicted for various samples using the 'model solid' approach with the above two assumptions.

Large differences are seen between the profiles generated using the model solid approach with the two different assumptions for the band line-up prior to the consideration of strain effects. For the compressive strained well the interpolation of the $\mathrm{E}_{v, a v}$ values leads to a shallow light hole well whilst the assumption of an unstrained $\Delta \mathrm{E}_{c} / \Delta \mathrm{E}_{g}=0.4$ leads to a shallow electron well. The value of $\Delta \mathrm{E}_{c} / \Delta \mathrm{E}_{g}=0.52 \pm 0.05$ estimated from the data of E863 is closer to the band line-up predicted by the interpolated $E_{v, a v}$ values. This is supported by photocurrent data from E863, which show the E1-HH1 exciton holding up better under applied bias than would be expected for the very shallow electron well predicted in column (b) of Table 1. For the tensile strained samples both assumptions lead to a decreasing heavy hole offset, which should lead to reduced power saturation, but this is a much larger effect using the assumption of an unstrained band offset. The positions of the E1-LH1 and E1-HH1 levels are calculated as a function of strain, for band profiles generated using the two assumptions described above. In both cases a good fit to Fig. 1(b) is obtained, showing that the energies of these two transitions are not particularly dependent on the band offsets.

\section{Conclusions.}

It is shown that moderate strains can be advantageous for In GaAsP electro-absorption modulators. Optical data has enabled an estimate of the band offset ratio for a lattice matched and a strain balanced structure InGaAsP/InGaAsP MQW. A model is developed to describe the changes in band offsets as strain is introduced, with the results being very sensitive to the assumptions made in determining the band line-up prior to including the effects of strain.

This work was supported by the U.K. government LINK program on 'MOCVD of III/V Materials for Integration', which is jointly funded by the SERC and DTI.

\section{References.}

1. E.T. Yu, J.O. McCaldin, and T.C. McGill, in Solid State Physics Vol 46 (Academic, San Diego, 1993).

2. S.L. Wong, R.J. Nicholas, C.G. Cureton, J.M. Jowett, and E.J. Thrush. Semicond. Sci. Technol. 7 (1992) 493.

3. C.G. Van de Walle. Phys. Rev. B39 (1989) 1871.

4. M.P.C.M. Krijn. Semicond. Sci. Technol. 6 (1991) 27.

5. M. Silver. private communication 